\title{
Adiabatic divergence of the chaotic layer width and acceleration of chaotic and noise-induced transport
}

\author{
S.M. Soskin ${ }^{\mathrm{a}, \mathrm{b}}$, R. Mannella ${ }^{\mathrm{c}}$, O.M. Yevtushenko ${ }^{\mathrm{d}}$ \\ ${ }^{a}$ Institute of Semiconductor Physics, Pr. Nauki 45, Kiev, 03028, Ukraine \\ ${ }^{\mathrm{b}}$ Abdus Salam ICTP, Strada Costiera 11, Trieste, 34100, Italy \\ ${ }^{\mathrm{c}}$ Dipartimento di Fisica, Università di Pisa, Largo Pontecorvo 3, Pisa, 56127, \\ Italy \\ ${ }^{d}$ Physics Department, Ludwig-Maximilians-Universität München, München, \\ D-80333, Germany
}

\begin{abstract}
We show that, in spatially periodic Hamiltonian systems driven by a time-periodic coordinate-independent (AC) force, the upper energy of the chaotic layer grows unlimitedly as the frequency of the force goes to zero. This remarkable effect is absent in any other physically significant systems. It gives rise to the divergence of the rate of the spatial chaotic transport. We also generalize this phenomenon for the presence of a weak noise and weak dissipation. We demonstrate for the latter case that the adiabatic AC force may greatly accelerate the spatial diffusion and the reset rate at a given threshold.
\end{abstract}

Key words: Adiabatic, chaos, transport, noise, diffusion, threshold PACS: 05.45.Ac, 05.40.-a, 05.45.Pq., 66.30.-h

\section{Introduction}

The basic chaotic formation in perturbed Hamiltonian systems is [1-3] a chaotic layer associated with a separatrix of the unperturbed (integrable) Hamiltonian system. Even in the simplest case, when the unperturbed system is one-dimensional while the perturbation is time-periodic, both the transport within the layer $[4,5]$ and its structure in the Poincaré section $[3,5,6]$ possess very complicated features related to the homoclinic tangle. At the same time, the boundaries of the layer are well defined [3] as the last invariant curves 
which limit the layer (in the phase space) from above and below (in the energy scale), and can be found in numerical simulations [3]. For both theory and applications, one of the most important characteristics of the layer is its width in energy [1-3,7] or in related quantities [8-11]. It might be assumed that, if the perturbation is very weak, then the layer should necessarily be narrow. This natural assumption seemed to be supported by numerous examples (e.g. $[1-3,7-11])$. However, we have shown[12] that the situation may drastically differ in a rather general case, namely for any spatially periodic system driven by a slow $A C$ force (the number of dimensions may exceed 1). In this case, the upper energy boundary of the layer diverges as the frequency of the force goes to zero (Fig. 1). In Sec. 2, we explain this effect and present the theory, both within the separatrix map approach (for moderately small frequencies) and beyond this approach (for smaller frequencies). We also demonstrate that the spatial transport may be very fast in the adiabatic case, being chaotic on sufficiently long time-scales.

We briefly comment on the relation between our work and the study of the conventional adiabatic case [10]. It was shown in [10] that, for a system with adiabatically slowly pulsating parameters, the homoclinic tangle covers most of the range swept by the instantaneous separatrix. If the pulsation of parameters in [10] were weak, the range swept would be narrow in energy and, hence, the chaotic layer would be narrow too. Our system essentially differs from that considered in [10] (our perturbation is not parametric) so the result of [10] cannot be directly applied to it. If nevertheless the result of [10] were formally generalized to our system it would give that the homoclinic tangle in the adiabatic limit covered the whole phase space, thus hinting at a divergence of the chaotic layer width. It would still not describe the form of the divergence however.

The above features of the Hamiltonian chaotic dynamics suggest that the adiabatic AC-drive may strongly affect noise-induced phenomena in spatially periodic systems subject to weak dissipation and noise. In Sec. 3, we discuss this for the spatial diffusion and for the reset rate at a given threshold of the kinetic energy.

\section{Hamiltonian systems}

An archetypal example [1] of an AC-driven spatially periodic system is a pendulum (Fig. 2) driven by a weak single-harmonic AC force:

$$
\begin{aligned}
& \dot{q}=p, \quad \dot{p}=-d U_{0} / d q-h \omega_{0}^{2} \sin \left(\omega_{f} t\right) \equiv-d U / d q \\
& U_{0} \equiv U_{0}(q)=-\omega_{0}^{2} \cos (q)
\end{aligned}
$$



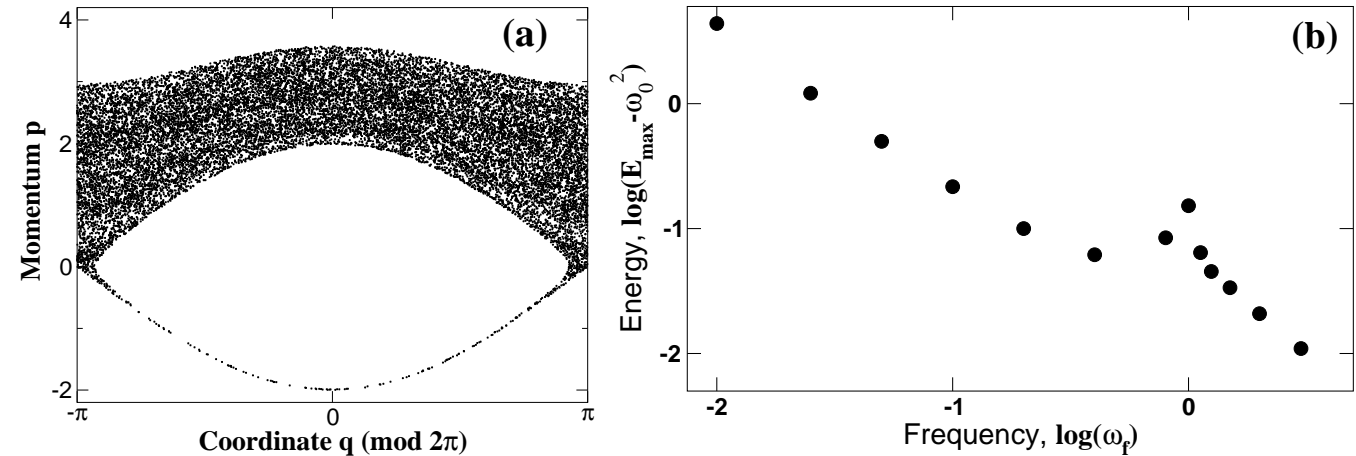

Fig. 1. (a). The trajectory in the stroboscopic (for $t=n 2 \pi / \omega_{f}$ with $n=0,1,2, \ldots$ ) Poincaré section for the system (1) with $\omega_{0}=1, h=0.01, \omega_{f}=0.01$ and initial conditions $(p(0)=0, q(0)=\pi)$. Number of points is 20000. We map all points onto the interval $[-\pi, \pi[: q \rightarrow q-2 \pi[(q+\pi) /(2 \pi)]$. This mapping does not affect the energy $E \equiv p^{2} / 2+U_{0}(q)$ and, hence, neither the chaotic layer width. (b). Spectral dependence of the maximum $E_{\max }$ of the energy $E$ in the chaotic layer $\left(\omega_{0}=1, h=0.01\right)$.
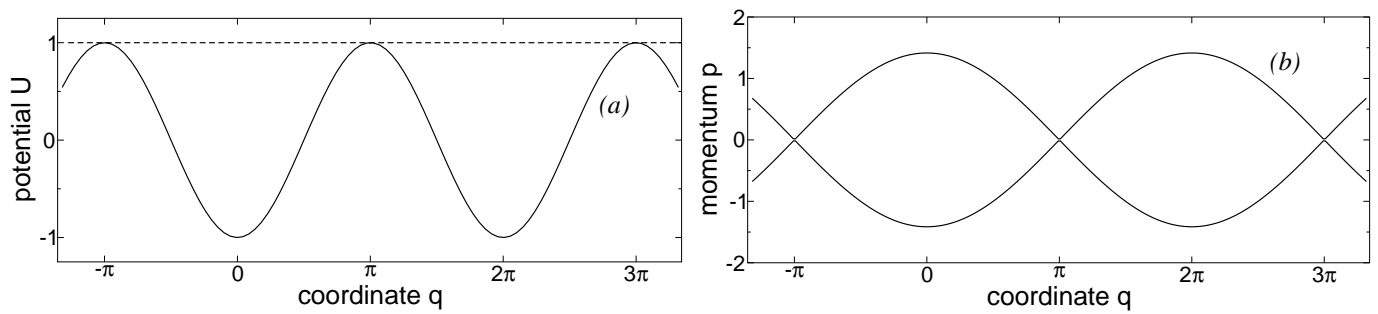

Fig. 2. Potential (in (a)) and separatrix (in (b)) for the pendulum (1) with $\omega_{0}=1$.

$$
U \equiv U(q, t)=U_{0}(q)+q h \omega_{0}^{2} \sin \left(\omega_{f} t\right), \quad h \ll 1 .
$$

\subsection{Separatrix map}

One of the conventional approaches for the treatment of Hamiltonian chaos is the separatrix map [1-3]. For the system (1), the map reads as (cf. [1]):

$$
\begin{aligned}
E_{i+1}= & E_{i}+\sigma_{i} \delta \sin \left(\varphi_{i}\right), \\
\varphi_{i+1}= & \varphi_{i}+\frac{\omega_{f} \pi\left(3+\operatorname{sign}\left(E_{i+1}-E_{s}\right)\right)}{2 \omega\left(E_{i+1}\right)}, \\
\sigma_{i+1}= & \sigma_{i} \operatorname{sign}\left(E_{i+1}-E_{s}\right), \quad\left|\sigma_{i}\right|=1, \\
& \delta=\frac{2 \pi h \omega_{0}^{2}}{\cosh \left(\frac{\pi \omega_{f}}{2 \omega_{0}}\right)}, \quad E_{s}=\omega_{0}^{2},
\end{aligned}
$$

where $E_{s}$ is the separatrix energy while $\omega(E)$ is the frequency of the eigenoscil- 
lation of the momentum for a given energy $E$.

Conventional heuristic criteria [1-3] for the onset of chaos in the map (2) are invalid for small frequencies, i.e. for $\omega_{f} \ll \omega_{0}$. We analyze the onset of chaos in the map in a different way.

First of all, we use the fact that any state at the separatrix (i.e. with $E=E_{s}$ ) belongs to the chaotic trajectory. Indeed, the angle $\varphi$ in such a state is not correlated with the angle at the previous iteration of the map since $\omega\left(E_{s}\right)=0$. The variable $\sigma$ in the separatrix state is not correlated to that at the previous iteration of the map either. Thus, we choose $E_{0}=E_{s}$ and an arbitrary $\varphi_{0} \neq$ $n \pi$. Being interested in the upper boundary of the chaotic layer, we choose $\sigma_{0}=\operatorname{sign}\left(\sin \left(\varphi_{0}\right)\right)$, so that $E_{1}-E_{0}>0$.

Let $\omega_{f}$ be small, so that $\varphi_{n+1}-\varphi_{n} \ll 2 \pi$. In the relevant asymptotic limit $h \rightarrow 0$, this condition is satisfied for

$$
\omega_{f} \ll \omega_{0} / \ln (1 / h)
$$

Then, the map (2) reduces to the system of differential equations:

$$
\begin{aligned}
& \frac{\mathrm{d} E}{\mathrm{~d} t}=\frac{\omega(E)}{2 \pi} \operatorname{sign}\left(\sin \left(\varphi_{0}\right)\right) \delta \sin (\varphi), \\
& \frac{\mathrm{d} \varphi}{\mathrm{d} t}=\omega_{f}, \\
& E(0)=E_{s}, \quad \varphi(0)=\varphi_{0} \neq n \pi, \quad n=0, \pm 1, \pm 2, \ldots
\end{aligned}
$$

The system (4) describes the chaotic trajectory only until $E$ returns to the immediate vicinity of $E_{s}$. After that, the angle correlation is again lost $[13,14]$. In between the passages near the separatrix, the trajectory $E(\varphi)$ can be explicitly found from (4) in terms of the action variable $I$ :

$$
\begin{aligned}
& I-I_{s} \equiv \int_{E_{s}}^{E} \mathrm{~d} \tilde{E} \frac{1}{\omega(\tilde{E})}=\operatorname{sign}\left(\sin \left(\varphi_{0}\right)\right) \frac{\delta}{2 \pi \omega_{f}}\left(\cos \left(\varphi_{0}\right)-\cos (\varphi)\right), \\
& \operatorname{sign}\left(\sin \left(\varphi_{0}\right)\right)\left(\cos \left(\varphi_{0}\right)-\cos (\varphi)\right)>0 .
\end{aligned}
$$

The upper boundary of the chaotic layer in phase space, $E_{c l}^{(u)}(\varphi)$, corresponds to the value of $\varphi_{0}$ for which $I(\varphi)$ is larger than that for any other $\varphi_{0}$. This corresponds to $\varphi_{0} \rightarrow+0$, so that $E_{c l}^{(u)}(\varphi)$ and the maximum energy on the boundary are given respectively as 


$$
\begin{aligned}
& I\left(E_{c l}^{(u)}\right)-I_{s}=\frac{\delta}{2 \pi \omega_{f}}(1-\cos (\varphi)), \\
& I\left(E_{\text {max }}\right)-I_{s}=\frac{\delta}{\pi \omega_{f}} .
\end{aligned}
$$

For all spatially periodic systems driven by the AC force, the adiabatic limit of $\delta$ is not equal to zero (see e.g. Eq. (2), relevant for the case (1)), unlike other physically significant systems ${ }^{1}$ (in particular, the archetypal model of the pendulum driven by a wave [1-3,8-11]; cf. also the recent study [15] of the width of the chaotic layer for the pendulum driven by two waves running in opposite directions). Therefore $E_{\max }(7)$ diverges in the adiabatic limit. The physical origin of this divergence is the accumulation of the energy gain during many iterations of the map.

The separatrix map (2) is valid only if $E_{\max }-E_{s} \ll E_{s}$ i.e. only for

$$
\omega_{f} \gg h \omega_{0}
$$

but, still, the adiabatic divergence of $E_{\max }$ for the separatrix map suggests that there should be a divergence of $E_{\max }$ for the original Hamiltonian system too. The latter divergence should be described differently however.

\subsection{Hamiltonian dynamics}

The separatrix map approach was generalized in [12] for the whole adiabatic range (3), where $E_{\max }-E_{s}$ may be $\stackrel{\sim}{>} E_{s}$. We present here just the result for $E_{\max }$, which is described as the solution of the transcendental equation in terms of the elliptic integral of the second order $\mathrm{E}(x)$ :

$$
\begin{aligned}
& \frac{\mathrm{E}(x)}{\sqrt{x}}=1+\frac{\pi h}{2} \frac{\omega_{0}}{\omega_{f}}, \quad x \equiv\left(1+\frac{E_{\max }-E_{s}}{2 E_{s}}\right)^{-1}, \\
& \mathrm{E}(x) \equiv \int_{0}^{\pi / 2} \mathrm{~d} \varphi \sqrt{1-x^{2} \sin ^{2}(\varphi)} .
\end{aligned}
$$

The agreement with the results of simulations is excellent (Fig. 3). Note also that $E_{\text {max }}(9)$ matches the separatrix map result (7) in the range of the validity of the latter i.e. for $h \omega_{0} \ll \omega_{f} \ll \omega_{0} / \ln (1 / h)$.

$\overline{1}$ There may be some other examples with non-zero adiabatic limit of $\delta$ but they seem to be of a mathematical interest only. 


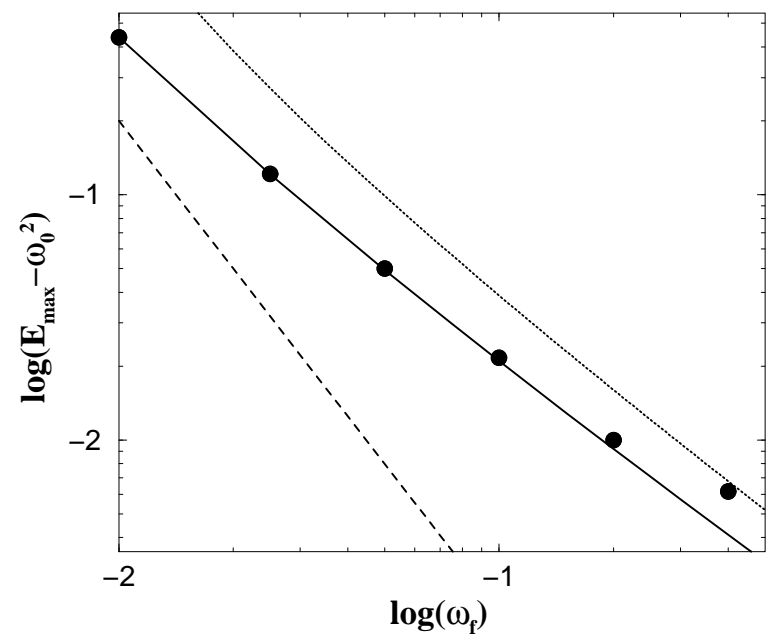

Fig. 3. Spectral dependence of the layer maximum energy for the system (1) with $\omega_{0}=1$ and $h=0.01$ : circles, solid, dashed and dotted lines correspond respectively to simulations, Eq. (9) and its asymptotes for small and large values of $\omega_{f} /\left(h \omega_{0}\right)$.

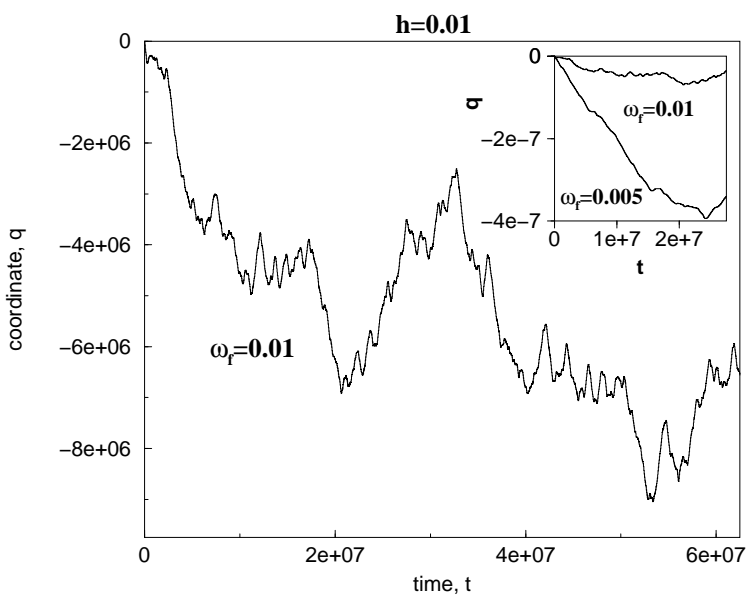

Fig. 4. A typical trajectory of the system (1) with $\omega_{0}=1, h=0.01, \omega_{f}=0.01$ on a large time-scale. The inset compares the trajectories for $\omega_{f}=0.01$ and $\omega_{f}=0.005$.

To get an insight into the mechanisms of the transport in the chaotic layer, we consider the frequency range corresponding to the most pronounced divergence of the layer width,

$$
\omega_{f} \ll h \omega_{0} .
$$

To that end, we use a different approach. In the range (10), $E_{\max }-E_{s} \gg$ $E_{s} \equiv \omega_{0}^{2}$. Therefore, the term $U_{0}(q)$ in $U(q)$ in (1) may be neglected for the major part of the chaotic trajectory between subsequent passages near the separatrix. Then the equations of motion reduce to those of a free particle driven by a time-periodic force. Their solution reads 


$$
\begin{aligned}
& q(t)=A+\left(B-\frac{h \omega_{0}^{2}}{\omega_{f}}\right) t+\frac{h \omega_{0}^{2}}{\omega_{f}^{2}} \sin \left(\omega_{f} t\right), \\
& p(t) \equiv \dot{q}=B-\frac{h \omega_{0}^{2}}{\omega_{f}}\left(1-\cos \left(\omega_{f} t\right)\right),
\end{aligned}
$$

where $A=q(0)$ and $B=p(0)$.

For the initial state $(p(0)=0, q(0)=\pi)$ (which corresponds to the saddle of the original unperturbed system $\left.H_{0}=p^{2} / 2+U_{0}(q)\right)$, the integration constant $B$ is equal to 0 so that the velocity (11) oscillates from 0 to $-2 h \omega_{0}^{2} / \omega_{f}$ while the kinetic energy $\mathcal{K} \equiv p^{2} / 2$ oscillates from 0 to

$$
\mathcal{K}_{\max }=2 h^{2} \omega_{0}^{4} / \omega_{f}^{2}
$$

Of course, this result coincides with $E_{\max }-\omega_{0}^{2}(9)$ taken in the asymptotic limit of small $\omega_{f} /\left(h \omega_{0}\right)$.

The chaotization occurs when the system returns close to the separatrix. But, unlike the range (8) where the chaotization occurs relatively quickly (just for one iteration of the separatrix map), the chaotization in case (10) develops very slowly through small random-like shifts of $B$ occurring when the trajectory passes near the separatrix. The rigorous treatment is complicated (cf. the treatment of the chaotic layer and the chaotic diffusion for the conventional adiabatic case [8-11]). Here we briefly review the qualitative analysis developed in [12].

It is shown in $[12]$ that the quantity $B$ diffuses in the interval $\left[B_{\min }, B_{\max }\right]$ where the boundaries are approximated as

$$
B_{\min }=0, \quad B_{\max }=\frac{2 h \omega_{0}}{\omega_{f}}
$$

Chaotic trajectories generally spend most of the time close to the boundaries of regions of regular motion [5]. In our case, it means that, for most of the time, $B$ is close to either $B_{\min }$ or $B_{\max }$, i.e. the trajectory moves close to either the upper border of the chaotic layer, with average velocity $v \approx h \omega_{0}^{2} / \omega_{f}$, or the lower border, with average velocity $-v$. We call such regimes acceleration-braking flights, distinguishing them from the regime of a relatively fast diffusion across the layer. The duration $t_{f}$ of the flight may be estimated from the analysis of the diffusion of $B$ near the boundary of the layer. The diffusion constant for $B$ may be roughly estimated as $D_{B} \sim\left\langle(\Delta B)^{2}\right\rangle /\left(2 \pi / \omega_{f}\right)$ where $\left\langle(\Delta B)^{2}\right\rangle$ is the average squared change of $B$ at the end of the first period of perturbation (the first period is counted from an instant when $B=0$ ). It can be shown to be $\left\langle(\Delta B)^{2}\right\rangle \sim B_{\max }^{2}\left(\omega_{f} \Delta t_{1}\right)^{4}$ where $\Delta t_{1}$ is a typical small shift of the start of 
the second acceleration round with respect to the start of the second period of perturbation: $\Delta t_{1} \sim \omega_{0}^{-1} \ln \left(\omega_{0} /\left(h \omega_{f}\right)\right) \ll \omega_{f}^{-1}$. Therefore,

$$
t_{f} \sim \frac{B_{\max }^{2}}{D_{B}} \sim \omega_{0}^{-1} \frac{\left(\omega_{0} / \omega_{f}\right)^{5}}{\ln ^{4}\left(\omega_{0} /\left(h \omega_{f}\right)\right)} .
$$

Basing on numerical studies, it has been suggested in [16] that the spatial chaotic diffusion reduces in the adiabatic limit to the normal type, i.e. $\langle(q(t)-$ $\left.q(0))^{2}\right\rangle=D t^{2}$. Assuming the validity of this, we estimate the diffusion constant $D$ as the ratio between the squared length of the flight $l_{f}^{2}$ and its duration $t_{f}$ :

$$
D \sim \frac{l_{f}^{2}}{t_{f}} \equiv v^{2} t_{f} \sim \omega_{0} h^{2} \frac{\left(\omega_{0} / \omega_{f}\right)^{7}}{\ln ^{4}\left(\omega_{0} /\left(h \omega_{f}\right)\right)}
$$

Thus, our analysis provides for intuitive arguments in favor of a strong acceleration of the spatial chaotic transport as $\omega_{f} \rightarrow 0$, and results of the numerical integration support our semi-quantitative estimates (Fig. 4). It is a challenging problem however to study this issue rigorously (cf. for example [11] for the study of the diffusion of the adiabatic invariant in the conventional case).

Note also that on a very coarse time scale $t \gg t_{f}$ we observe a slow average drift (cf. Fig. 4) the direction and absolute value of which depend on initial conditions. Its explanation is a challenging problem for future.

Finally, we note that the action ${ }^{2} I \equiv(2 \pi)^{-1} \oint p \mathrm{~d} q$ conventionally [8-11] chosen as the lowest-order adiabatic invariant, is not conserved for motion above the barrier in our system: on the major part of a trajectory, $K \gg \omega_{0}^{2}$ and hence $I \approx p$ while $|p|$ varies in a wide range (from 0 to $\sim h \omega_{0}^{2} / \omega_{f}$, for the chaotic trajectory). The correct lowest-order adiabatic invariant for our system is

$$
\tilde{I}=I+\frac{h \omega_{0}^{2}}{\omega_{f}}\left(1-\cos \left(\omega_{f} t\right)\right) .
$$

To the lowest order in $\left(h \omega_{0} / \omega_{f}\right)$, the quantity $\tilde{I}$ coincides with the integration constant $B$ of (11) on the major part of the trajectory ${ }^{3}$. So, the chaotic layer width in $\tilde{I}$ is the same as in $B$, i.e. equal to $B_{\max } \equiv 2 h \omega_{0}^{2} / \omega_{f}$, diverging as $\omega_{f} \rightarrow 0$.

$\overline{2}$ For the motion above the barrier, $\oint$ means the integration over a relevant $2 \pi$ range of $q$.

3 Small changes of $\tilde{I}$ occur only when the separatrix is approached (cf. the conventional case [8-11]). 


\section{Noise-driven underdamped systems}

A strong acceleration of chaotic transport in the adiabatic limit suggests that similar mechanisms may be relevant in the presence of weak noise and dissipation. Consider the following equations of motion

$$
\begin{aligned}
& \dot{q}=p, \quad \dot{p}=-d U_{0} / d q-\Gamma p-h \omega_{0}^{2} \sin \left(\omega_{f} t\right)+f_{n}(t), \\
& \left\langle f_{n}(t)\right\rangle=0, \quad \quad\left\langle f_{n}(t) f_{n}(0)\right\rangle=2 \Gamma T \delta(t), \\
& \Gamma \ll \omega_{0}, \quad h \ll 1, \quad T \ll \Delta U \equiv 2 \omega_{0}^{2} .
\end{aligned}
$$

There is a broad literature devoted to studies of models like (17) (see e.g. $[17,18,7]$ and references therein). However, to the best of our knowledge, there were only two papers $[19,20]$ devoted to the acceleration of the noise-induced spatial diffusion in underdamped spatially periodic systems by means of the adiabatic ac-drive while the operation of threshold devices linked to a model like (17) was studied only in our recent paper [20]. We briefly review these issues below.

\subsection{Spatial diffusion}

First, let us discuss the diffusion in the case without ac-drive $(h=0)$. The characteristic time-scale is the mean escape time from the well [17],

$$
t_{e s c} \sim \Gamma^{-1} \frac{T}{\Delta U} \exp (\Delta U / T) .
$$

On larger time-scales, the spatial diffusion is well developed $[17,21]$ i.e. nearly all systems have escaped from the initially populated well and are distributed over many wells. The diffusion rate is characterized by the diffusion constant $D$. The latter may be estimated as [21]

$$
D \sim \frac{\left\langle l^{2}\right\rangle}{t_{e s c}}
$$

where $\left\langle l^{2}\right\rangle$ is the mean-square jump length.

In the two-dimensional case, diffusion analogous to the aforementioned type ${ }^{4}$ is used in some technological processes [18], and it may be important to accel-

$\overline{4}$ It is called [18] the surface diffusion in this case. 
erate it by a weak ${ }^{5}$ perturbation. It was suggested in [19] (and more recently in $[12,20])$ to use a low-frequency ac-drive for this aim. We have demonstrated the validity of this idea by means of computer simulations for the one-dimensional underdamped case (cf. also [19]). The diffusion greatly accelerates as the frequency of the $\mathrm{AC}$ force decreases provided it is not too small: if it becomes smaller than some optimal frequency $\omega_{f}^{(o p t)} \sim t_{e s c}^{-1}$, then the diffusion slows down. The existence of the optimal frequency was noticed in [19] too but its theoretical estimate was incorrect.

\subsection{Threshold devices}

Threshold devices are devices which are reset to an initial state if a given dynamical variable becomes larger than a preset threshold. Such devices, linked to various stochastic systems, were considered in the context of stochastic resonance (see e.g. [22]). We suggest to use such devices in a different context: the adiabatic ac-drive in spatially periodic systems may strongly affect the mean rate of resets.

Consider e.g. a threshold in the kinetic energy $K_{t h}$. Let it be higher than the barrier height $\Delta U$ and let the temperature $T$ be smaller than both $\Delta U$ and $K_{t h}-\Delta U$. In the absence of the ac-drive, the mean rate of resets has the following activation-like dependence on $T$ :

$$
R \propto \exp \left(-\frac{K_{t h}}{T}\right), \quad h=0, \quad T \ll K_{t h} .
$$

If the ac-drive is on, then the system starting from the top of the barrier may follow the deterministic trajectory along which it may reach a high kinetic energy. Though chaos is irrelevant in this case, the deterministic trajectories coincide with the regular-like trajectories described above in the context of chaos. This leads to a significant decrease of the activation energy. For example, if $K_{t h}<K_{\max } \equiv E_{\max }+\omega_{0}^{2}$, then the activation energy reduces just to $\Delta U$ :

$$
\begin{aligned}
& R \propto \exp \left(-\frac{\Delta U}{T}\right), \quad \frac{\omega_{f}}{\omega_{0}} \stackrel{\sim}{<} h \ll 1, \\
& \Delta U<K_{t h}<K_{\max } \equiv E_{\max }+\omega_{0}^{2}, \quad T \ll \Delta U, K_{t h}-\Delta U .
\end{aligned}
$$

Thus, even a rather weak adiabatic ac-drive drastically increases the rate. Such a strong sensitivity of the reset rate to the presence of the adiabatic ac-drive

5 A strong perturbation should be avoided as it may destroy the surface itself. 
may be used for control or as a new kind of sensors. The relevant circuit may be based e.g. on an underdamped Josephson junction [23].

\section{Conclusions}

It has been shown that the class of Hamiltonian systems defined as spatially periodic systems subject to coordinate-independent time-periodic force, possesses a unique property. In such a system, the width of the chaotic layer and the transport rate within the layer diverge as the frequency of the force goes to zero. This occurs due to the presence in the chaotic trajectory of long acceleration periods followed by braking periods after which the trajectory returns close to the separatrix where small irregular changes of the adiabatic invariant occur.

If a spatially periodic system is subject to a weak noise and a weak dissipation, then a coordinate-independent adiabatic time-periodic force may greatly accelerate the noise-induced spatial diffusion and increase the reset rate in threshold devices.

\section{References}

[1] G.M. Zaslavsky, R.D. Sagdeev, D.A. Usikov and A.A. Chernikov, Weak Chaos and Quasi-Regular Patterns, Cambridge University Press, Cambridge, 1991.

[2] A.J. Lichtenberg and M.A. Liebermann, Regular and Stochastic Motion, Springer, New York, 1992.

[3] G.M. Zaslavsky, Physics of Chaos in Hamiltonian systems, Imperial Colledge Press, London, 1998.

[4] V. Rom-Kedar, Nonlinearity 7, 441 (1994).

[5] G.M. Zaslavsky, Phys. Rep. 371, 461 (2002).

[6] A.I. Neishtadt, V.V. Sidorenko, D.V. Treschev, Chaos 7, 2 (1997).

[7] S.M. Soskin, R. Mannella and P.V.E. McClintock, Phys. Rep. 373, 247 (2003).

[8] A.I. Neishtadt, Sov. J. Plasma Phys. 12, 568 (1986).

[9] J.R. Cary, D.F. Escande, J.L. Tennyson, Phys. Rev. A 34, 4256 (1986).

[10] Y. Elskens and D.F. Escande, Nonlinearity 4, 615 (1991).

[11] D.L. Bruhwiler and J.R. Cary, Physica D 40, 265 (1989). 
[12] S.M. Soskin, O.M. Yevtushenko, R. Mannella, Phys. Rev. Lett. 95, 224101 (2005).

[13] S.M. Soskin, O.M. Yevtushenko, R. Mannella, Phys. Rev. E 77, 036221 (2008).

[14] S.M. Soskin, O.M. Yevtushenko, R. Mannella, in Chaos, Complexity and Transport: Theory and Applications, PROCS 7837, eds. C. Chandre, X. Leoncini, and G. Zaslavsky (World Scientific, Singapore, in press); see also arXiv:0711.4827.

[15] I.I. Shevchenko, Phys. Lett. A 372, 808 (2008).

[16] R. Harrish, S. Rajasekar, K.P.N. Murthy, Phys. Rev. E 65, 046214 (2002).

[17] V.I. Mel'nikov, Phys. Rep. 209, 1 (1991).

[18] T. Ala-Nissila, R. Ferrando, S.C. Ying, Adv. Phys. 51, 949 (2002).

[19] J. Kallunki, M. Dube, T. Ala-Nissila, Surface Science 460, 39 (2000).

[20] S.M. Soskin, O.M. Yevtushenko and R. Mannella, in Noise and Fluctuations in Circuits, Devices and Materials, eds. M. Macucci, L.K.J. Vandamme, C. Ciofi, M.B. Weissman, Proceedings of SPIE Vol. 6600 (2007), article 660008.

[21] R. Ferrando, R. Spadacini, G.E. Tommei, Phys. Rev. E 48, 2437 (1993).

[22] N.G. Stocks, Phys. Rev. Lett. 84, 2310 (2000).

[23] A. Barone and G. Paterno, Physics and Applications of the Josephson Effect, Wiley, New York, 1982. 